\title{
Resilience to chronic mild stress-induced anhedonia preserves the ability of the ventral hippocampus to respond to an acute challenge
}

\section{Paola Brivio}

University of Milan: Universita degli Studi di Milano

\section{Maria Teresa Gallo}

University of Milan: Universita degli Studi di Milano

\section{Piotr Gruca}

Polish Academy of Sciences: Polska Akademia Nauk

\section{Magdalena Lason}

Polish Academy of Sciences: Polska Akademia Nauk

\section{Ewa Litwa}

Polish Academy of Sciences: Polska Akademia Nauk

\section{Fabio Fumagalli}

University of Milan: Universita degli Studi di Milano

\section{Mariusz Papp}

Polish Academy of Sciences: Polska Akademia Nauk

Francesca Calabrese ( $\sim$ francesca.calabrese@unimi.it)

Università degli Studi di Milano: Universita degli Studi di Milano https://orcid.org/0000-0001-7946-2424

\section{Research Article}

Keywords: chronic stress, acute stress, IEGs, ERGs, resilience

Posted Date: February 16th, 2022

DOI: https://doi.org/10.21203/rs.3.rs-1349468/v1

License: (c) (i) This work is licensed under a Creative Commons Attribution 4.0 International License. Read Full License 


\section{Abstract}

Stress is one of the main precipitating factors for psychiatric disorders and its effects may depend on its duration and intensity. Interestingly, the ability to react to stressful life events may alter the response to a subsequent novel stressor. However, there are differences in the individual susceptibility to stress, with some individuals displaying vulnerability and others showing resistance.

Hence, we investigated whether the vulnerability and resilience to the chronic mild stress (CMS) paradigm, in terms of hedonic phenotype, may alter the ability to cope with a novel acute restraint stress by focusing on neuroplastic mechanisms within the ventral (vHip) and dorsal (dHip) hippocampus, the amygdala (Amy) and the prefrontal cortex (Pfc) of adult male rats. At molecular level, we found that the resilience to CMS was critical to preserving the ability to face a novel challenge measured as neuronal activation. Indeed, the resilient phenotype positively correlated with the enhancement of early response genes specifically in vHip. These results highlight the role of this hippocampal subregion for the development of therapeutic strategies to promote resilience against stress.

\section{Introduction}

Coping with stress involves the regulation of different systems, namely neuroendocrine, autonomic, metabolic, immune, and cardiovascular. The ability to efficiently adapt to daily experiences is defined as resilience, i.e. the capacity of an individual to face negative, social, psychosocial, and biological consequences of chronic stress that would otherwise compromise their general well-being [1].

At preclinical level, the chronic mild stress (CMS) animal model is largely employed to mimic the variability in the stress response characteristic of the human being [2] and to understand the phenomena set in motion in stressresilient individuals [3]. Indeed, CMS is a well-established procedure that allows stratifying the animals into two populations based on their hedonic behavior, one that is efficiently able to cope with the negative effects of stress, the stress-resilient subset, and the other that, instead, suffers from the negative consequences of the stress thus developing the pathological phenotype.

Even if resilience is now considered an active process [4], research aimed at understanding the underlying molecular mechanisms is, nowadays, sparce. On this topic, we, recently found that resilience to CMS, assessed as the absence of anhedonic-like symptoms, can be partly due to the activation of neuroplastic mechanisms within the ventral hippocampus, through the enhancement of the beta isoform of the glucocorticoid receptor [5].

To move a step forward, here, we decided to evaluate whether the response to a chronic stress experience may alter the brain's ability to react appropriately to a subsequent novel stressor. To this aim, rats that showed vulnerability or resilience to CMS, measured by means of the sucrose consumption test, were exposed to one hour of acute restraint stress (ARS) and sacrificed one hour later. Notably, since it has been observed that the response to an acute challenge may be altered if the type of stress has been presented previously [6, 7], ARS was not included as a stressor of the CMS protocol.

Finally, to study whether chronic stress may induce long-term effects and alter the capacity to react to novel challenges, we allowed vulnerable rats to recover from stress before exposing them to ARS.

On these bases, at molecular level, we focused on the expression of genes promptly induced by acute stimuli, the immediate early genes (IEGs) Arc and Cfos as well as on the expression of the early response genes (ERGs) Gadd45b, Sgk1, Dusp 1, Nr4a1 [8-12]. The reason why we decided to focus our attention on these genes relies on the evidence 
that they have been used as markers of neuronal activity for the study of neuronal circuits involved in several brain functions [13].

Our analyses were performed in the hippocampus (ventral vHip and dorsal dHip), the amygdala (Amy), and the prefrontal cortex (Pfc), limbic structures that mediate the emotional response and that support, through dynamic processes, the neuroplastic mechanisms that allow coping with external challenges. Indeed, the plasticity of these brain areas is fundamental to mediate the mechanisms of adaptation following acute stress exposure and chronic stressful-life events may alter the way through which these brain regions may respond to negative experiences [14].

\section{Material And Methods}

\section{Animals}

Adult male Wistar rats (Charles River, Germany) were brought into the laboratory one month before the start of the experiment. Except for the first 10 days after arrival when the animals were housed in groups of 10, they were single housed in standard laboratory conditions: food and water was freely available on a 12-h light/dark, constant temperature $\left(22 \pm 2^{\circ} \mathrm{C}\right)$ and humidity $(50 \pm 5 \%)$. All procedures used in this study have conformed to the rules and principles of the 2010/63 European Communities Council Directive and have been approved by the Local Bioethical Committee at the Maj Institute of Pharmacology, Polish Academy of Sciences, Krakow, Poland. All efforts were made to minimize animal suffering, to reduce the number of animals used and the animal studies comply with the ARRIVE guidelines.

\section{Stress procedure and behavioral test}

After adaptation to the housing conditions, rats were trained to consume $1 \%$ sucrose solution as previously described [5] and sucrose consumption was monitored at weekly intervals during the whole experiment.

The experimental paradigm was depicted in Fig. 1A. Briefly, rats were randomly divided into two matched groups: one group was subjected to the chronic mild stress (CMS) procedure for a period of 2 consecutive weeks (see: [15] for details) and the other one was not subjected to the stress protocol. After 2 weeks of CMS, we separated animals showing the anhedonic phenotype (CMS-vul) from the animals that were resilient to the stress protocol (CMS-res). At this time point, CMS-vulnerable group was further divided into two subgroups and part of these animals were left undisturbed in their home cages until the full recovery of the behavioral phenotype (CMS-vul + recovery). Moreover, half of all the experimental groups were exposed to one hour of acute restraint stress (ARS), a novel type of stressor to whom animals were not subjected during the CMS procedure.

The animals of naïve groups were decapitated 24 hours after the final sucrose test whereas the animals of the ARS groups were sacrificed one hour after the ARS procedure. vHip, dHip, Amy, and Pfc were dissected from the whole brain according to the plates Paxinos and Watson [16] for the subsequent molecular analysis.

\section{Blood collection and corticosterone measurement}

After the sacrifice, trunk blood was collected in EDTA tubes and centrifuged for 20 min at $3000 \mathrm{~g}$ at $4^{\circ} \mathrm{C}$ for the separation of the plasma. Corticosterone plasma levels were assessed with the IBL ELISA kit.

RNA preparation and gene expression analysis by quantitative Real-time PCR 
Total RNA was isolated using PureZol RNA isolation reagent (Bio-Rad Laboratories, Italy) and quantified by spectrophotometric analysis as previously described [17]. An aliquot of each sample was treated with DNase (ThermoFisher scientific, Italy) to avoid DNA contamination. Real-time polymerase chain reaction (RT-PCR) was performed to assess Arc, Cfos, Gadd45b, Sgk1, Dusp1, Nr4a1 mRNA levels. RNA was analyzed by TaqMan qRT-PCR instrument (CFX384 real time system, Bio-Rad Laboratories, Italy) using the iScriptTM one-step RT-PCR kit for probes (Bio-Rad Laboratories, Italy) (see [18] for details). Samples were run in 384 well formats in triplicate as multiplexed reactions with the normalizing internal control 36B4 (the primers and probes sequences are listed in Table1). A comparative cycle threshold $(\mathrm{Ct})$ method was used to calculate the relative target gene expression.

Table 1

A) Sequences of forward and reverse primers and probes used in Real-time PCR analyses and purchased from EurofinsMWG-Operon. B) Probes purchased from Life Technologies, which did not disclose the sequences.

\begin{tabular}{|c|c|c|c|}
\hline A) Gene & Forward primer & Reverse primer & Probe \\
\hline Arc & GGTGGGTGGCTCTGAAGAAT & ACTCCACCCAGTTCTTCACC & GATCCAGAACCACATGAATGGG \\
\hline Cfos & TCCTTACGGACTCCCCAC & CTCCGTTTCTCTTCCTCTTCAG & TGCTCTACTTTGCCССTTCTGCC \\
\hline Sgk1 & GGTGGGTGGCTCTGAAGAAT & ACTCCACCCAGTTCTTCACC & GATCCAGAACCACATGAATGGG \\
\hline Dusp1 & TGTGCCTGACAGTGCAGAAT & ATCTTTCCGGGAAGCATGGT & АTCCTGTCCTTCCTGTACCT \\
\hline $36 b 4$ & TCAGTGCCTCACTCCATCAT & AGGAAGGCCTTGACCTTTTC & TGGATACAAAAGGGTCCTGG \\
\hline B) Gene & Accession number & Assay ID & \\
\hline Gadd45b & ВС085337.1 & Rn01452530_gl & \\
\hline $\mathrm{Nr} 4 \mathrm{a} 1$ & BC097313.1 & Rn01533237_m1 & \\
\hline
\end{tabular}

\section{Z-score}

Z score for each gene has been calculated in the single animals according to the formula

Zscore $=\frac{X-\mu}{\sigma}$

$\mathrm{X}$ represents the fold change of each animal while $\mu$ and $\sigma$ represent the mean and the standard deviation of the No stress/Naïve group.

Z activation was then calculated by averaging the Z score of each gene in vHip, dHip, Amy, Pfc.

\section{Statistical analysis}

The results of the SCT were analyzed with the one-way or two-way analysis of variance (ANOVA) with repeated measures, followed by Fisher's Protected Least Significant Difference (PLSD), whereas the molecular analyses with the two-way ANOVA with PLSD. The results of the two-way ANOVA are listed in the supplementary tables 1-2-3-4. Each experimental group consists of 8 rats. Significance for all tests was assumed for $p<0.05$. Data are presented as means \pm standard error (SEM).

\section{Results}




\section{3 weeks of rest from CMS leads to a complete recovery of the behavioral phenotype}

As we previously observed [5], 2 weeks of CMS induced the development of vulnerable and resilient phenotypes, as indicated by the significant reduction of sucrose intake after $1(-56 \%, p<0.001$ vs No stress) and $2(-68 \%, p<0.001$ vs No stress) weeks of CMS only in a subgroup of stressed animals that were, therefore, named vulnerable (CMS-vul) (Fig. 1B).

Vulnerable animals took 3 weeks to fully recover from the pathological phenotype (Fig. 1C). Indeed, we observed that starting from the first week after the end of CMS exposure, animals showed a statistically significant increase in the sucrose intake ( 1 week: $+60 \%, \mathrm{p}<0.001$ vs CMS-vul/2weeks; 2 weeks: $+57 \%, \mathrm{p}<0.001$ vs CMS-vul/2weeks) and, interestingly, 3 weeks of recovery from stress were enough to completely recover from the anhedonic phenotype (+ $148 \%, p<0.001$ vs CMS-vul/2weeks). Accordingly, at the last time point, sucrose intake of the CMS-vul + recovery group was not statistically different from the baseline, whereas, after 1 and 2 weeks of recovery, sucrose intake was significantly lower in comparison to the vulnerable group at the beginning of the experiment (week 1 recovery: $-38 \%$, $p$ $<0.001$ vs CMS-vul/baseline; week2 recovery: -40\%, $p<0.001$ vs CMS-vul/baseline).

The IEGs Arc and Cfos are upregulated by ARS independently from the behavioral phenotype.

To map the neuronal responsiveness of brain areas involved in the acute stress response, we measured the expression levels of the immediate early genes Arc and Cfos in vHip, dHip, Amy and Pfc.

We observed that, in the vHip of non-stressed animals, both Arc and Cfos were increased by ARS (Arc: $+33 \%, p<0.01$ vs No stress/Naive; Cfos: $+404 \%$, $p<0.001$ vs No stress/Naive). Of note, regardless of the response to CMS in term of hedonic phenotype and of the period of rest, both the IEGs were upregulated by ARS in CMS-vul (Arc: $+48 \%$, p < 0.001 vs CMS-vul/Naive; Cfos: $+121 \%, p<0.001$ vs CMS-vul/Naive), in CMS-res (Arc: $+34 \%, p<0.05$ vs CMSres/Naive; Cfos: $+154 \%$, $p<0.001$ vs CMS-res/Naive) and in CMS-vul + recovery (Arc: $+56 \%$, $p<0.001$ vs CMS-vul + recovery/Naive; Cfos: $+92 \%$, p $<0.001$ vs CMS-vul + recovery /Naive) (Fig. 2A-B).

Similarly, we observed the same effect due to ARS in all the experimental conditions in the dHip, Amy and Pfc (Table 2A-B-C).

These results indicate that the exposure to 2 weeks of CMS did not alter the ability of the brain regions considered to react with a novel challenging condition. 
Table 2

Analysis of Arc and Cfos mRNA levels in the dorsal hippocampus (dHip) (A), the amygdala (Amy) (B) and the prefrontal cortex (PfC) (C) of chronically stressed rats exposed to 1 hour of acute restraint stress (ARS).

\begin{tabular}{|c|c|c|c|c|c|c|c|c|c|}
\hline \multirow[t]{2}{*}{ IEGs } & & \multicolumn{2}{|c|}{ No stress } & \multicolumn{2}{|c|}{ CMS VUL } & \multicolumn{2}{|c|}{ CMS RES } & \multicolumn{2}{|c|}{ CMS VUL-REC } \\
\hline & & Naive & ARS & Naive & ARS & Naive & ARS & Naive & ARS \\
\hline \multirow{4}{*}{$\begin{array}{l}\text { A } \\
\text { dHip }\end{array}$} & Arc & $100 \pm 8$ & $152 \pm 3 * \star \star \star$ & $94 \pm 6$ & $151 \pm 8$ \#\#\# & $114 \pm 10$ & $159 \pm 11$ §§§ & $127 \pm 10$ & $162 \pm 10$ \\
\hline & & & & & & & & * \# & $\wedge \wedge$ \\
\hline & Cfos & $100 \pm 16$ & $470 \pm 34 * \star \star *$ & $202 \pm 40$ & $475 \pm 80$ & $189 \pm 15$ & $422 \pm 25$ & $207 \pm 20$ & $437 \pm 57$ \\
\hline & & & & & \#\#\# & & §§§ & & $\wedge \wedge \wedge$ \\
\hline \multirow{4}{*}{$\begin{array}{l}\text { B } \\
\text { Amy }\end{array}$} & Arc & $100 \pm 9$ & $226 \pm 11$ & $102 \pm 9$ & $236 \pm 18$ & $131 \pm 11$ & $234 \pm 17$ & $126 \pm 16$ & $220 \pm 17$ \\
\hline & & & $\star \star * *$ & & \#\#\# & & §§§ & & $\wedge \wedge \wedge$ \\
\hline & Cfos & $100 \pm 19$ & $451 \pm 43$ & $93 \pm 12$ & $444 \pm 29$ & $161 \pm 14$ & $473 \pm 21$ & $186 \pm 23$ & $419 \pm 38$ \\
\hline & & & $\star * *$ & & \#\#\# & & §§§ & *\# & $\wedge \wedge \wedge$ \\
\hline \multirow{4}{*}{$\begin{array}{l}\text { C } \\
\text { Pfc }\end{array}$} & Arc & $100 \pm 18$ & $245 \pm 35$ & $103 \pm 14$ & $251 \pm 15$ & $209 \pm 25$ & $321 \pm 7$ & $143 \pm 16$ & $259 \pm 26$ \\
\hline & & & $\star \star \star$ & & \#\#\# & 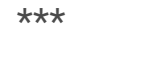 & $\S \S$ & & ${ }^{\wedge \wedge}$ \\
\hline & Cfos & $100 \pm 26$ & $161 \pm 24$ & $111 \pm 13$ & $201 \pm 17$ & $186 \pm 25$ & $298 \pm 34$ & $222 \pm 22$ & $265 \pm 23$ \\
\hline & & & & & \#\# & $\star \star \#$ & $\S \S$ & $\star \star \star \# \#$ & \\
\hline
\end{tabular}

The data are the mean \pm SEM: ${ }^{*} p<0.05,{ }^{* *} p<0.01,{ }^{* * *} p<0.001$ vs No stress/Naive; ${ }^{\#} p<0.05,{ }^{\# \#} p<0.01,{ }^{\# \# \#} p<0.001$

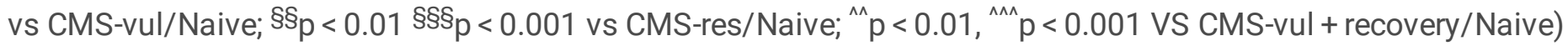
(two-way ANOVA, Fisher's PLSD).

Corticosterone plasma levels are affected by chronic and acute stress exposure.

Given the fundamental role of the HPA axis activity in stress response [19], we measured corticosterone plasma levels and, as previously demonstrated [5], we observed that CMS elevated levels of circulating CORT selectively in vulnerable animals $(+88 \%, p<0.05$ vs No stress/Naïve), whereas this modulation was observed neither in resilient nor in vulnerable + recovery groups. Moreover, in line with the notion that acute stressor leads to an enhancement of the HPA axis activity, we found that ARS increased levels of CORT in non-stressed animals ( $+100 \%, p<0.05$ vs No stress/Naïve) as well as in resilient rats $(+76 \%, p>0.05$ vs No stress/Naïve) even if the augmentation in the latter group did not reach the statistical significance probably due to the complexity of the experimental settings (Fig. 3 ).

The results obtained highlight that the negative effects of chronic stress led to a disruption of the negative feedback that regulates the activity of the HPA axis specifically in vulnerable animals; at the same time, these results indicate that the ability of the HPA axis to properly respond to novel challenging condition, as it occurs in healthy subjects, is maintained also in animals resilient to CMS.

The ability of the vHip to deal with a novel challenge in terms of ERGs is preserved selectively in resilient animals.

Seen the effects on CORT levels, we decided to measure whether differences in its release could influence the transcription of the ERGs, also known as glucocorticoid responsive genes, namely Gadd45b, Sgk1, Dusp1 and Nr4a1, in a brain region-specific manner. 
In vHip, we found that Gadd45b mRNA levels (Fig. 4A) were enhanced by ARS in non-stressed (+ 58\%, p $<0.001$ vs No stress/Naïve), in CMS-res (+ 20\%, p < 0.05 vs CMS-res/Naïve), in CMS-vul + recovery $(+40 \%, p<0.001$ vs CMS-vul + recovery /Naïve) groups but not in CMS-vul animals. Similarly, ARS induced a similar modulation of Sgk1 and Dusp1 (Fig. 4B-C respectively), with their mRNA levels being upregulated in unstressed (Sgk1: $+84 \%, p<0.001$ vs No stress/Naïve; Dusp1: $+61 \%$, $p<0.001$ vs No stress/Naïve), in resilient (Sgk1: $+40 \%, p<0.05$ vs CMS-res/Naïve; Dusp1: $+73 \%, \mathrm{p}<0.001$ vs CMS-res/Naïve) as well as in recovery group (Sgk1: +42\%, $\mathrm{p}<0.05$ vs CMS-vul + recovery/ Naïve; Dusp1: $+36 \%$, $p<0.001$ vs CMS-vul + recovery/Naïve), whereas this effect was completed blunted in vulnerable animals.

Differently, as shown in Fig. 4D, we observed that Nr4a1 gene expression was increased by ARS regardless of the behavioral phenotype (No stress: $+45 \%$, $p<0.001$ vs No stress/Naïve; CMS-vul: $+38 \%, p<0.001$ vs CMS-vul/Naïve; $+30 \%, p<0.001$ vs CMS-res/Naïve; $+40 \%, p<0.001$ vs CMS-vul/Naïve).

By contrast, we observed an overall upregulation of the ERGs expression due to the acute challenge in all the experimental settings in dHip, Amy and Pfc, as shown in Table 3A-B-C. Of note, in Pfc we found that ERGs were increased under basal conditions in resilient animals in comparison to non-stressed group (Table 3C).

These results suggest that the vHip seems to be the brain region mostly affected by CMS in the ability to modulate neuronal plasticity following a novel stressor. 
Table 3

Analysis of Gadd45b, Sgk1, Dusp1 and Nr4a1 mRNA levels in the dorsal hippocampus (dHip) (A), the amygdala (Amy) (B) and the prefrontal cortex (PfC) (C) of chronically stressed rats exposed to 1 hour of acute restraint stress (ARS).

\begin{tabular}{|c|c|c|c|c|c|c|c|c|c|}
\hline \multirow[t]{2}{*}{ ERGs } & & No stress & \multicolumn{2}{|c|}{ CMS VUL } & \multicolumn{2}{|c|}{ CMS RES } & \multicolumn{3}{|c|}{ CMS VUL-REC } \\
\hline & & Naive & ARS & Naive & ARS & Naive & ARS & Naive & ARS \\
\hline \multirow{8}{*}{$\begin{array}{l}\text { A } \\
\text { dHip }\end{array}$} & Gadd45b & $100 \pm 4$ & $144 \pm 3$ & $109 \pm 7$ & $134 \pm 5$ & $123 \pm 6$ & $141 \pm 5$ & $104 \pm 6$ & $144 \pm 8$ \\
\hline & & & $\star * \star$ & & \#\# & ** & $\S$ & $\S$ & $\wedge \wedge \wedge$ \\
\hline & Sgk1 & $100 \pm 2$ & $162 \pm 12$ & $97 \pm 5$ & $129 \pm 11$ & $93 \pm 3$ & $119 \pm 16$ & $93 \pm 6$ & $133 \pm 11$ \\
\hline & & & $\star \star *$ & & \# & & & & $\wedge \wedge$ \\
\hline & Dusp1 & $100 \pm 5$ & $158 \pm 10$ & $127 \pm 8$ & $145 \pm 9$ & $118 \pm 3$ & $151 \pm 7$ & $94 \pm 8$ & $136 \pm 5$ \\
\hline & & & $\star \star \star *$ & * & & & $\S \S$ & $\# \# \S$ & $\wedge \wedge \wedge$ \\
\hline & $\mathrm{Nr} 4 \mathrm{a} 1$ & $100 \pm 5$ & $135 \pm 6$ & $111 \pm 6$ & $133 \pm 7$ & $103 \pm 7$ & $144 \pm 8$ & $116 \pm 7$ & $144 \pm 8$ \\
\hline & & & ** & & \# & & §§§ & & $\wedge \wedge$ \\
\hline \multirow{8}{*}{$\begin{array}{l}\text { B } \\
\text { Amy }\end{array}$} & Gadd45b & $100 \pm 7$ & $170 \pm 8$ & $113 \pm 10$ & $166 \pm 5$ & $128 \pm 7$ & $165 \pm 9$ & $128 \pm 7$ & $158 \pm 10$ \\
\hline & & & $\star \star \star$ & & \#\#\# & * & §§ & * & $\wedge$ \\
\hline & Sgk1 & $100 \pm 6$ & $152 \pm 12$ & $103 \pm 6$ & $124 \pm 8$ & $106 \pm 4$ & $128 \pm 5$ & $97 \pm 6$ & $112 \pm 5$ \\
\hline & & & $* * *$ & & \# & & $\S$ & & \\
\hline & Dusp1 & $100 \pm 5$ & $168 \pm 9$ & $92 \pm 6$ & $166 \pm 10$ & $108 \pm 7$ & $150 \pm 9$ & $114 \pm 9$ & $136 \pm 7$ \\
\hline & & & $\star \star \star *$ & & \#\#\# & & §§§ & & \\
\hline & Nr4a1 & $100 \pm 8$ & $194 \pm 13$ & $91 \pm 8$ & $186 \pm 10$ & $111 \pm 5$ & $179 \pm 10$ & $104 \pm 8$ & $183 \pm 16$ \\
\hline & & & $* * *$ & & \#\#\# & & §§§ & & $\wedge \wedge \wedge$ \\
\hline \multirow{8}{*}{$\begin{array}{l}\mathrm{C} \\
\mathrm{PfC}\end{array}$} & Gadd45b & $100 \pm 10$ & $200 \pm 22$ & $96 \pm 14$ & $235 \pm 23$ & $144 \pm 10$ & $257 \pm 26$ & $139 \pm 12$ & $226 \pm 15$ \\
\hline & & & 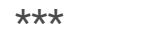 & & \#\#\# & *\# & §§§ & & ${ }^{\wedge \wedge}$ \\
\hline & Sgk1 & $100 \pm 4$ & $126 \pm 11$ & $92 \pm 3$ & $123 \pm 6$ & $111 \pm 7$ & $104 \pm 7$ & $103 \pm 4$ & $112 \pm 9$ \\
\hline & & & $\star \star$ & & \#\# & & & & \\
\hline & Dusp1 & $100 \pm 7$ & $149 \pm 14$ & $104 \pm 7$ & $154 \pm 15$ & $143 \pm 13$ & $150 \pm 13$ & $148 \pm 5$ & $153 \pm 11$ \\
\hline & & & * & & \#\#\# & * & & * & \\
\hline & Nr4a1 & $100 \pm 13$ & $271 \pm 20$ & $141 \pm 7$ & $263 \pm 28$ & $185 \pm 27$ & $263 \pm 19$ & $197 \pm 13$ & $277 \pm 35$ \\
\hline & & & $\star \star \star *$ & & \#\# & * & $\S$ & * & $\wedge \wedge$ \\
\hline
\end{tabular}

The data are the mean \pm SEM: ${ }^{*} p<0.05,{ }^{* \star} p<0.01,{ }^{* \star} p<0.001$ vs No stress/Naive; ${ }^{\#} p<0.05,{ }^{\# \#} p<0.01,{ }^{\# \#} p<0.001$ vs CMS-vul/Naive; $\S p<0.05, \S \S p<0.01, \S \S \S p<0.001$ vs CMS-res/Naive; ${ }^{\wedge} p<0.05,{ }^{\wedge \wedge} p<0.01,{ }^{\wedge \wedge} p<0.001$ VS CMSvul + recovery/Naive) (two-way ANOVA, Fisher's PLSD).

Z score activation of the ERGs highlights the role of vHip in the susceptibility to stress 
We calculated the $Z$ score activation in each brain region to get a combined overview of the modulation of the IEGs (Fig. 5A-B-C-D) and ERGs (Fig. 5E-F-G-H) following ARS.

We found a significant $Z$ activation of the IEGs due to ARS, regardless of the behavioral phenotype, in all the brain regions examined (Fig. 5A-B-C-D). Moreover, in Pfc (Fig. 5D), we observed an effect of the stress exposure per sè, with an increased $Z$ activation in resilient naïve animals in comparison to the non-stressed counterpart.

On the contrary, the $z$ activation of the ERGs highlighted that 2 weeks of CMS affected the ability of the vHip to deal with ARS (Fig. 5E) selectively in vulnerable animals. By contrast, in dHip (Fig. 5F), Amy (Fig. 5G), and Pfc (Fig. 5H), Z activation indicated that, despite stress exposure, these brain areas were not impaired in their ability to mount a stress response.

Finally, as observed for the IEGs (Fig. 5D), the significant Z activation of the ERGs in the Pfc (Fig. 5H) of resilient animals suggests that the $\mathrm{Pfc}$ activates mechanisms of resilience to face the negative effects of the CMS procedure.

Z score activation of the ERGs in vHip significantly correlates with the anhedonic phenotype

Finally, we performed the Pearson correlation analysis to figure out the relationship between the behavioral phenotype and the Z activation of the ERGs in vHip, dHip, Amy, and Pfc. Interestingly, as revealed by Person productmoment correlation coefficient analysis, the sucrose intake positively correlated with $Z$ activation of the ERGs specifically in vHip $\left(R^{2}=0.368, p<0.001\right)$ (Fig. 6A), but not in dHip $\left(R^{2}=0.056, p>0.05\right)\left(\right.$ Fig. 6B), Amy $\left(R^{2}=0.000, p\right.$ $>0.05$ ) (Fig. 6C) and in Pfc $\left(R^{2}=0.064, p>0.05\right)$ (Fig. 6D), thus strengthening the role of the ventral subregion of the hippocampus in the susceptibility to develop stress-related disorders.

\section{Discussion}

In this study, we demonstrated that animals that did not exhibit an anhedonic phenotype following 2 weeks of CMS preserve the ability of the ventral hippocampus to face a novel challenge, a function that is instead impaired in the subpopulation of animals showing the depressive-like behavior.

According to our previous results [5, 15], we confirm that anhedonia, a core symptom of depression in humans [20], was developed only in a part of animals exposed to the CMS paradigm. Indeed, approximately $30 \%$ of stressed animals displayed resilience to the stress protocol.

Moreover, in line with the finding that spontaneous recovery from the depressive-like behavior induced by 6 weeks of CMS was achieved after 4 weeks of rest [21], we showed that vulnerable animals took 3 weeks of washout to completely recover the pathological phenotype.

After the behavioral characterization in terms of hedonic phenotype, we investigated the response of vulnerable and resilient animals to a novel acute stressor by focusing on neuronal activation to explore whether previous stress exposure may have affected their ability to deal with a new challenge.

As of IEGs, we found that, regardless of stress exposure, ARS enhanced the expression of Arc and Cfos in all the limbic structures investigated, an effect supported by the cumulative Z score activation, implicating that CMS does not interfere with the degree of limbic activation and suggesting that the responsiveness of these brain regions was retained, also after chronic stress, to promote neuronal activation following external stimuli. In line, we previously found that both ARS and acute forced swim stress exposure [22-25], as well as cognitive demanding task [15], led to 
an overexpression of the IEGs in unstressed animals in different brain regions; notably, in the Pfc, such modulation was still present after a period of washout following 4 weeks of chronic restraint stress [25].

The hallmark of the stress response is the activation of the HPA axis and the effects of chronic and acute stressors on the axis activity are described by the inverted U-shaped dose-response curve of glucocorticoids [19]. According to the timeline of stress exposure and the modulation of the axis, here we found that the elevated levels of CORT, due to chronic stress, are related to the vulnerability to CMS, whereas resilient animals properly activate the HPA axis. Moreover, the positive effect of ARS was completely absent not only in vulnerable animals but also in chronically stressed rats that recovered from stress, indicating that CMS induced long-lasting effects that interfere with glucocorticoid activation.

Afterward, we measured the expression of a series of genes, Gadd45b, Sgk1, Dusp1, Nr4a1, which could be clustered as early response genes. Indeed, the ERGs are rapidly transcribed following acute challenges and respond to HPA axis activity [8-11].

In the vHip we found that Gadd45b, Sgk1, Dusp1 were enhanced by ARS not only in the control group but also in animals that were resilient to CMS. By contrast, we did not observe this upregulation in CMS-vul group, whereas the vulnerable rats that recovered from the pathological phenotype were still able to mount such response.

The $z$ score activation of the ERGs following ARS supports these results, by highlighting the direction of genes' regulation in the brain areas considered and strengthening the finding that chronic stress induced functional alterations in vHip in our experimental settings.

These results further support the concept that this brain subregion is extremely susceptible to CMS, compromising its ability to cope with challenging situations. Additionally, the fact that resilient rats exhibited a control-like response to ARS suggests that the activation of the vHip, in terms of ERGs, may be a strategy set in motion to promote the resilience processes. This is sustained by the positive correlation we found in vHip between the behavioral phenotype and the $Z$ activation of the ERGs merely in the hippocampal subregion that controls emotional responses to stress. Accordingly, we have recently demonstrated that the novel antidepressant drug vortioxetine targeted specifically the vHip and modulated neuronal plasticity following an acute stressor in a region-specific manner [24]. Furthermore, other mechanisms set in motion specifically in vHip may contribute to the stress susceptibility or to the promotion of resilience after stress exposure at adulthood such as neurogenesis [26, 27], the activation of the GRb-RACK1-Bdnf pathway [5] and also the mitochondrial activity.

Our analyses revealed that the ERG Nr4a1 was enhanced by ARS in all the experimental groups, including the vulnerable ones, in line with the notion that this gene is highly expressed when the levels of circulating corticosterone are elevated [9]. Similarly, as we recently found [5], 2 weeks of CMS induced elevated levels of CORT in the plasma of vulnerable animals possibly sustaining the $\mathrm{Nr} 4 \mathrm{a} 1$ overexpression we observed in susceptible rats.

As observed for the IEGs, we found that in dHip, Amy and Pfc ARS increased mRNA levels of all the ERGs considered, regardless of the behavioral susceptibility to stress, pointing out that these limbic structures were not impaired in their ability to be promptly activated following novel stimuli.

Of note, only in Pfc we noticed an upregulation of the IEGs and Gadd45b, Dusp1, Nr4a1 in resilient naïve animals in comparison to the unstressed naïve counterpart, possibly suggesting that the overexpression of these genes may contribute promoting the mechanisms of resilience within the Pfc. Consistent with this finding, it has been shown that mice resilient to the chronic predator or social defeat stress had a greater degree of Cfos expression in the 
glutamatergic neurons of the Pfc $[28,29]$, thus indicating that the enhanced neuronal activation in naïve animals might represent a pro-resilience adaptation. Actually, the Pfc has been associated with stressor resistance [30], and the results obtained with the $Z$ activation indeed emphasized the stress-coping strategies set in motion by the Pfc to promote resilience [31] through the enhancement of neuroplastic mechanisms.

Moreover, the physiological recovery from CMS at behavioral level was paralleled by a restored ability to deal with challenging conditions measured as proper response in terms of IEGs and ERGs following the novel acute stressor. Nevertheless, ARS did not enhance circulating levels of CORT in vulnerable animals following the recovery period, suggesting that the regulation of ERGs expression is complex and involves different and independent systems, which will be further investigated in future studies.

A potential limitation of our findings derives by the evidence that we primarily rely on mRNA data to infer changes in function, i.e. the ability to cope with a further challenge. However, we need to consider that the fast coping to the acute challenge needs to rely on evaluating changes in gene expression as initial responses whereas changes in the related protein levels would take much longer. Further, as our findings have been collected from male rats, we cannot say whether these results can also be generalized to female rats.

In summary, our results confirm the key role played by the vHip, the hippocampal subregion mainly involved in the regulation of emotion and in the management of the HPA axis activity [32], not only in the development of the anhedonic phenotype but also in the mechanisms of resilience set in motion in response to novel stress exposure. Ideally, the possibility to measure vHip activation in humans would provide useful indication about the potential effect exerted by stress exposure, and then be used as prognostic biomarkers.

Further, the findings here described suggest that development of pharmacological and non-pharmacological strategies able to selectively modulate the activity of these brain regions may be useful in promoting resilience.

\section{Declarations}

\section{Ethics approval}

All procedures used in this study have conformed to the rules and principles of the 2010/63 European Communities Council Directive and have been approved by the Local Bioethical Committee at the Maj Institute of Pharmacology, Polish Academy of Sciences, Krakow, Poland.

\section{Consent to participate}

Not applicable

\section{Consent for publication}

Not applicable

\section{Availability of data and materials}

The datasets generated during and/or analyzed during the current study are available from the corresponding author on reasonable request.

\section{Acknowledgments}


None

\section{Funding and disclosure}

The behavioral part of the study was supported by the statutory activity of the Maj Institute of Pharmacology Polish Academy of Sciences (Krakow, Poland) to M.P. This work was supported by a grant from MIUR Progetto Eccellenza (2018-2022) to the Department of Pharmacological and Biomolecular Sciences, University of Milan, Milan, Italy and from the Italian Ministry of University and Research (PRIN201719F) to F.C.

Competing Interest: The authors have no relevant financial or non-financial interests to disclose.

\section{Author contributions:}

Conceptualization: Paola Brivio, Francesca Calabrese; Investigation: Paola Brivio, Francesca Calabrese, Fabio Fumagalli; Methology: Paola Brivio, Maria Teresa Gallo, Piotr Gruca, Madgdalena Lason, Ewa Litwa, Mariusz Papp; Data Curation: Paola Brivio; Formal analysis: Paola Brivio, Francesca Calabrese; Writing original draft: Paola Brivio, Francesca Calabrese; Review and editing: Mariusz Papp, Fabio Fumagalli.

All the authors approved the final version of the manuscript.

\section{References}

1. Russo SJ, Murrough JW, Han M-H et al (2012) Neurobiology of resilience. Nat Neurosci 15:1475-1484. https://doi.org/10.1038/nn.3234

2. Willner P (2017) The chronic mild stress (CMS) model of depression: History, evaluation and usage. Neurobiol, Stress

3. Dziedzicka-Wasylewska M, Solich J, Korlatowicz A, Faron-Górecka A (2021) What Do the Animal Studies of Stress Resilience. Teach Us? Cells 10:1630. https://doi.org/10.3390/cells10071630

4. McEwen BS (2016) In pursuit of resilience: stress, epigenetics, and brain plasticity. Ann N Y Acad Sci 1373:5664. https://doi.org/10.1111/nyas. 13020

5. Brivio P, Buoso E, Masi M et al (2021) The coupling of RACK1 with the beta isoform of the glucocorticoid receptor promotes resilience to chronic stress exposure. Neurobiol Stress 100372.

https://doi.org/10.1016/j.ynstr.2021.100372

6. Nasca C, Zelli D, Bigio B et al (2015) Stress dynamically regulates behavior and glutamatergic gene expression in hippocampus by opening a window of epigenetic plasticity. Proc Natl Acad Sci 112:14960-14965. https://doi.org/10.1073/pnas.1516016112

7. Pastor-Ciurana J, Rabasa C, Ortega-Sánchez JA et al (2014) Prior exposure to repeated immobilization or chronic unpredictable stress protects from some negative sequels of an acute immobilization. Behav Brain Res 265:155-162. https://doi.org/10.1016/j.bbr.2014.02.028

8. Shipp LE, Lee JV, Yu C-Y et al (2010) Transcriptional Regulation of Human Dual Specificity Protein Phosphatase 1 (DUSP1) Gene by Glucocorticoids. PLoS ONE 5:e13754. https://doi.org/10.1371/journal.pone.0013754

9. Helbling JC, Minni AM, Pallet V, Moisan MP (2014) Stress and glucocorticoid regulation of NR4A genes in mice. J Neurosci Res. https://doi.org/10.1002/jnr.23366

10. Itani OA, Liu KZ, Cornish KL et al (2002) Glucocorticoids stimulate human sgk1 gene expression by activation of a GRE in its 5'-flanking region. Am J Physiol Metab 283:E971-E979. 
https://doi.org/10.1152/ajpendo.00021.2002

11. Ma DK, Jang MH, Guo JU et al (2009) Neuronal activity-induced Gadd45b promotes epigenetic DNA demethylation and adult neurogenesis. Science 80. https://doi.org/10.1126/science.1166859

12. Fowler T, Sen R, Roy AL (2011) Regulation of Primary Response Genes. Mol Cell 44:348-360. https://doi.org/10.1016/j.molcel.2011.09.014

13. Guzowski JF, Timlin JA, Roysam B et al (2005) Mapping behaviorally relevant neural circuits with immediateearly gene expression. Curr Opin Neurobiol 15:599-606. https://doi.org/10.1016/j.conb.2005.08.018

14. Fuchs E, Flügge G (2003) Chronic social stress: effects on limbic brain structures. Physiol Behav 79:417-427. https://doi.org/10.1016/S0031-9384(03)00161-6

15. Calabrese F, Brivio P, Gruca P et al (2017) Chronic Mild Stress-Induced Alterations of Local Protein Synthesis: A Role for Cognitive Impairment. ACS Chem Neurosci 8:817-825. https://doi.org/10.1021/acschemneuro.6b00392

16. Paxinos G, Watson C (2007) The Rat Brain in Stereotaxic Coordinates Sixth Edition. Elsevier Acad Press

17. Brivio P, Homberg JR, Riva MA, Calabrese F (2019) Alterations of Glutamatergic Markers in the Prefrontal Cortex of Serotonin Transporter Knockout Rats: A Developmental Timeline. Cell Mol Neurobiol 39:715-720. https://doi.org/10.1007/s10571-019-00673-9

18. Sbrini G, Brivio P, Fumagalli M et al (2020) Centella asiatica L. Phytosome Improves Cognitive Performance by Promoting Bdnf Expression in Rat Prefrontal Cortex. Nutrients 12:355. https://doi.org/10.3390/nu12020355

19. McEwen BS, Bowles NP, Gray JD et al (2015) Mechanisms of stress in the brain. Nat Neurosci 18:1353-1363. https://doi.org/10.1038/nn.4086

20. American Psychiatric Association (2013) Diagnostic and statistical manual of mental disorders (5th ed.). Washington, DC

21. Alves ND, Correia JS, Patrício P et al (2017) Adult hippocampal neuroplasticity triggers susceptibility to recurrent depression. Transl Psychiatry 7:e1058-e1058. https://doi.org/10.1038/tp.2017.29

22. Marchisella F, Paladini MS, Guidi A et al (2020) Chronic treatment with the antipsychotic drug blonanserin modulates the responsiveness to acute stress with anatomical selectivity. Psychopharmacology 237:17831793. https://doi.org/10.1007/s00213-020-05498-9

23. Brivio P, Sbrini G, Riva MA, Calabrese F (2020) Acute Stress Induces Cognitive Improvement in the Novel Object Recognition Task by Transiently Modulating Bdnf in the Prefrontal Cortex of Male Rats. Cell Mol Neurobiol 40:1037-1047. https://doi.org/10.1007/s10571-020-00793-7

24. Brivio P, Corsini G, Riva MA, Calabrese F (2019) Chronic vortioxetine treatment improves the responsiveness to an acute stress acting through the ventral hippocampus in a glucocorticoid-dependent way. Pharmacol Res 142:14-21. https://doi.org/10.1016/j.phrs.2019.02.006

25. Brivio P, Sbrini G, Corsini G et al (2020) Chronic Restraint Stress Inhibits the Response to a Second Hit in Adult Male Rats: A Role for BDNF Signaling. Int J Mol Sci 21:6261. https://doi.org/10.3390/ijms21176261

26. Levone BR, Cryan JF, O'Leary OF (2015) Role of adult hippocampal neurogenesis in stress resilience. Neurobiol, Stress

27. Anacker C, Luna VM, Stevens GS et al (2018) Hippocampal neurogenesis confers stress resilience by inhibiting the ventral dentate gyrus. Nature 559:98-102. https://doi.org/10.1038/s41586-018-0262-4

28. Covington HE, Lobo MK, Maze I et al (2010) Antidepressant Effect of Optogenetic Stimulation of the Medial Prefrontal Cortex. J Neurosci 30:16082-16090. https://doi.org/10.1523/JNEUROSCI.1731-10.2010

Page 13/19 
29. Adamec R, Toth M, Haller J et al (2012) A comparison of activation patterns of cells in selected prefrontal cortical and amygdala areas of rats which are more or less anxious in response to predator exposure or submersion stress. Physiol Behav 105:628-638. https://doi.org/10.1016/j.physbeh.2011.09.016

30. Sinha R, Lacadie CM, Constable RT, Seo D (2016) Dynamic neural activity during stress signals resilient coping. Proc Natl Acad Sci 113:8837-8842. https://doi.org/10.1073/pnas.1600965113

31. Maier SF, Watkins LR (2010) Role of the medial prefrontal cortex in coping and resilience. Brain Res 1355:52-60. https://doi.org/10.1016/j.brainres.2010.08.039

32. Gulyaeva NV (2019) Functional Neurochemistry of the Ventral and Dorsal Hippocampus: Stress, Depression, Dementia and Remote Hippocampal Damage. Neurochem Res 44:1306-1322. https://doi.org/10.1007/s11064018-2662-0

\section{Figures}

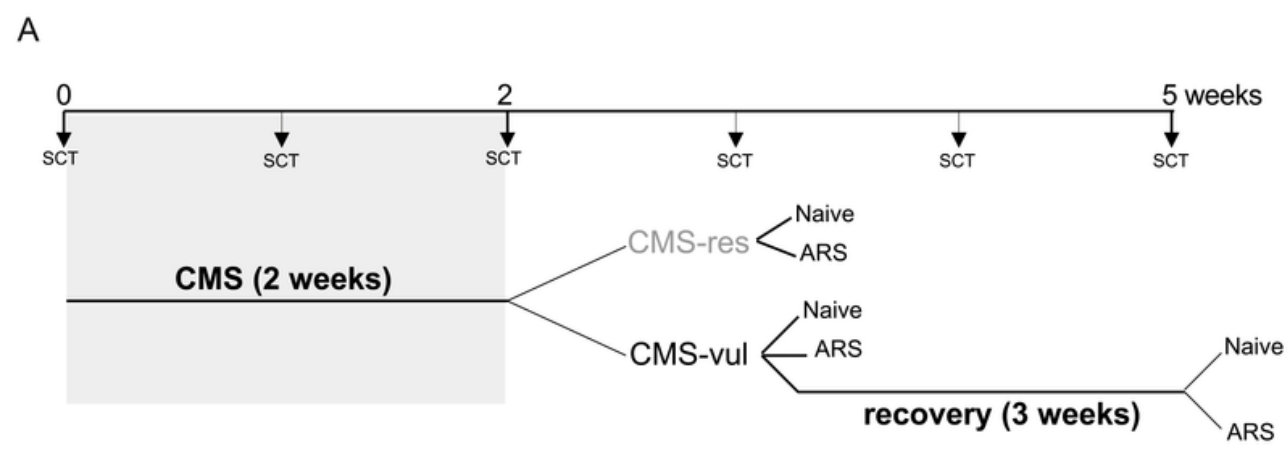

B

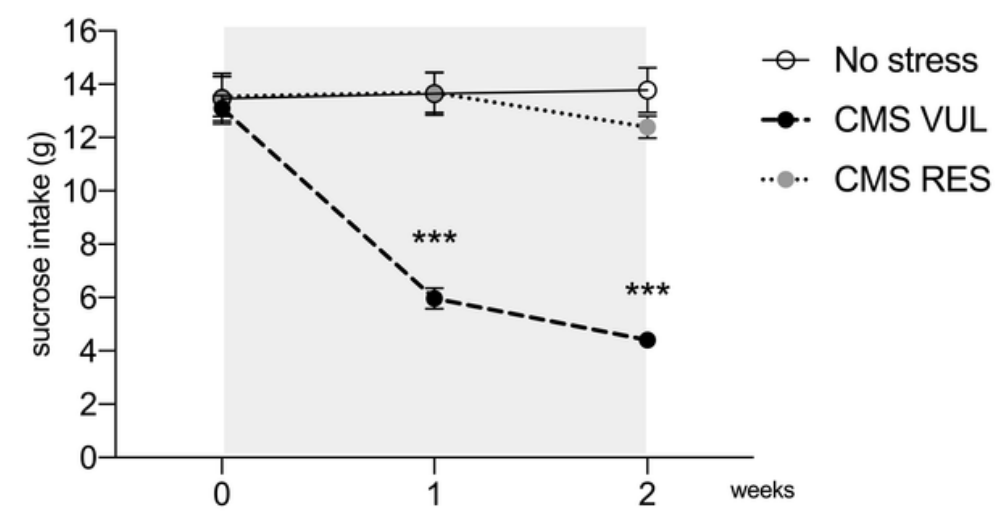

C

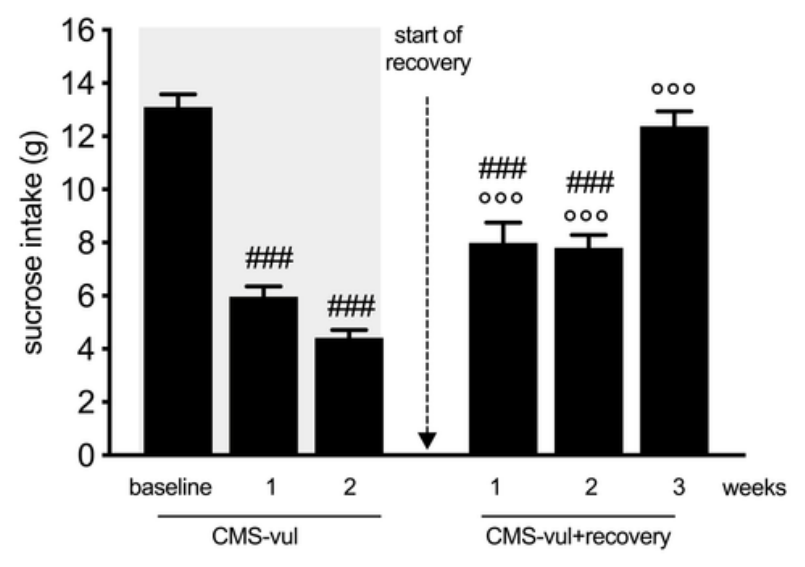

\section{Figure 1}

Sucrose consumption test in animals exposed to chronic mild stress (CMS) after a period of recovery from stress.

Panel A: experimental paradigm; panel B: sucrose consumption test (SCT) was performed at weekly intervals during the CMS procedure; Panel C: SCT performed in vulnerable animals after three weeks of recovery from CMS. Data are the mean \pm SEM: ${ }^{\star \star *} p<0.001$ vs No stress; ${ }^{\# \# ~} p<0.001$ vs CMS-vul-baseline, ${ }^{\circ 00}<0.001$ vs CMS VUL-week2 (one-way ANOVA, Fisher's PLSD). 


\section{vHip}

A

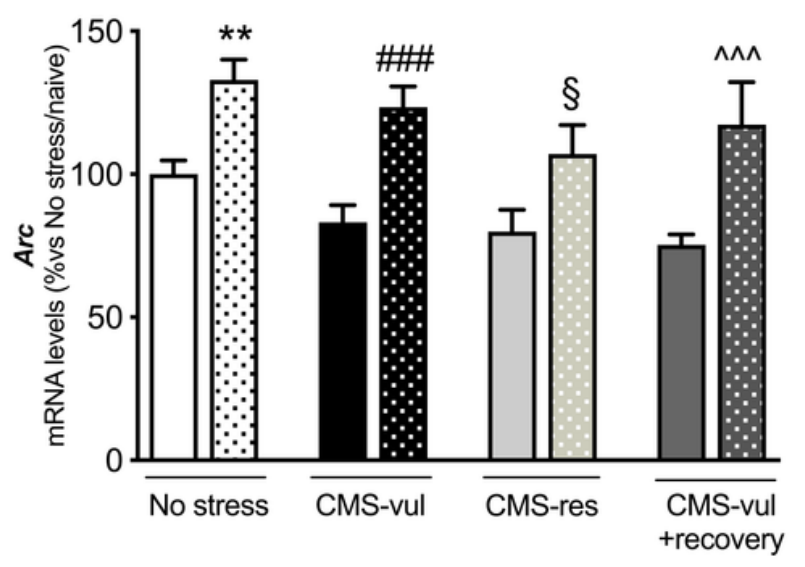

B

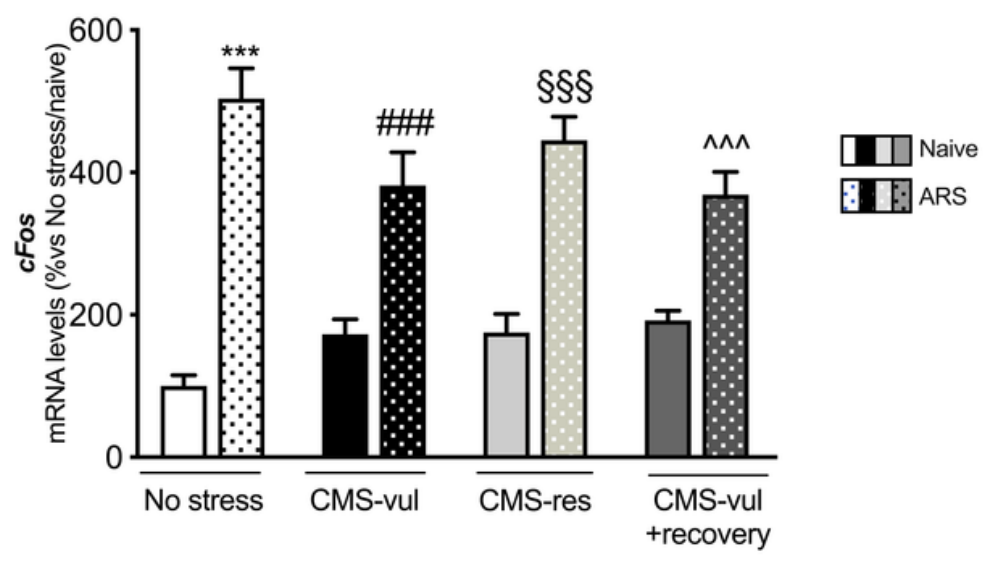

\section{Figure 2}

Analysis of $A r c$ and Cfos mRNA levels in the ventral hippocampus (vHip) of chronically stressed rats exposed to 1 hour of acute restraint stress (ARS).

The data are the mean \pm SEM: ${ }^{* \star} p<0.01,{ }^{\star \star \star} p<0.001$ vs No stress/Naive; ${ }^{\# \# \# ~} p<0.001$ vs CMS-vul/Naive; $\S^{\S}<0.05$, $\S \S \varsigma_{p}<0.001$ vs CMS-res/Naive; ${ }^{\wedge \wedge} p<0.001$ vs CMS-vul+recovery/Naive) (two-way ANOVA, Fisher's PLSD). 


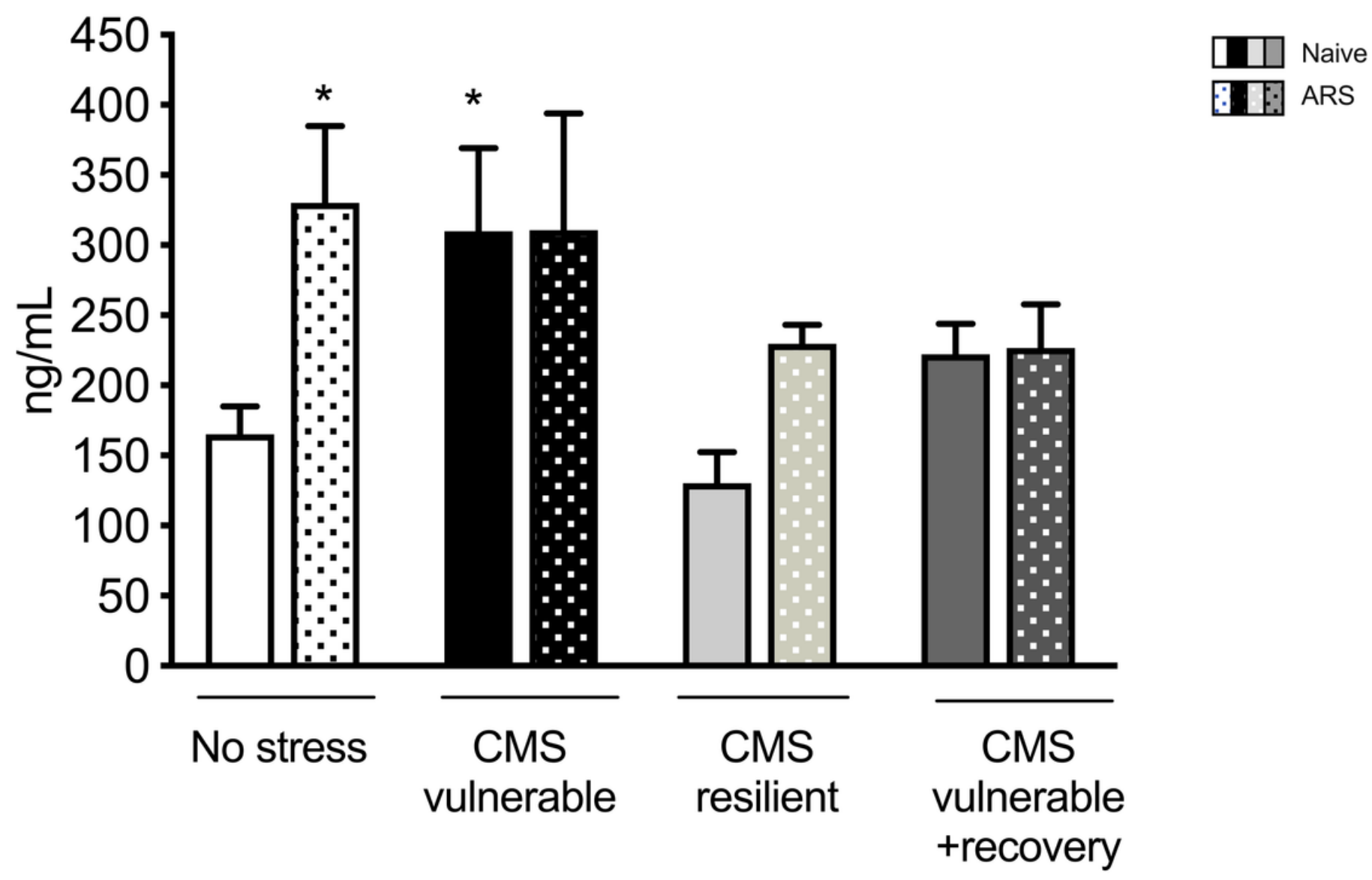

Figure 3

Analysis of corticosterone plasma levels in chronically stressed rats exposed to 1 hour of acute restraint stress (ARS).

The data are the mean \pm SEM: ${ }^{*} p<0.01$ vs No stress/Naïve (two-way ANOVA, Fisher's PLSD). 


\section{vHip}

A

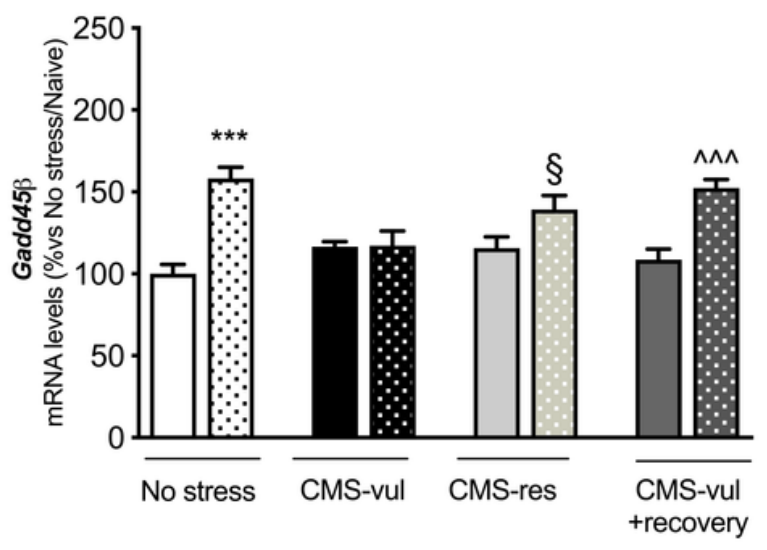

C

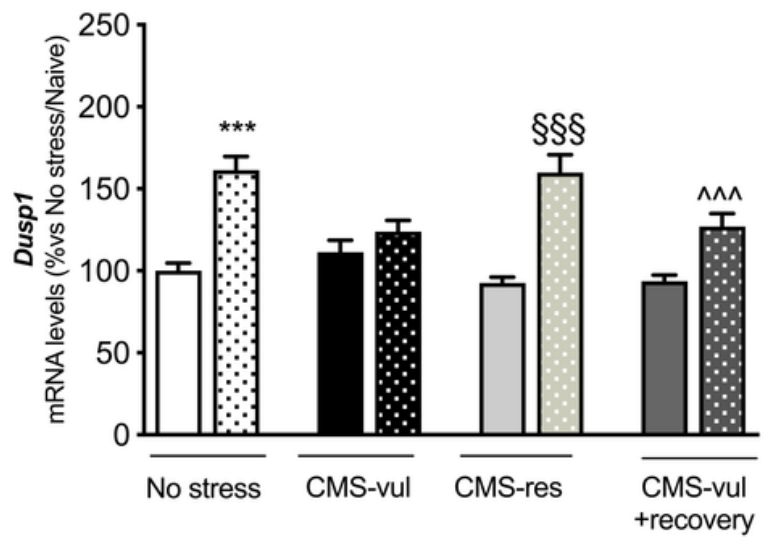

B

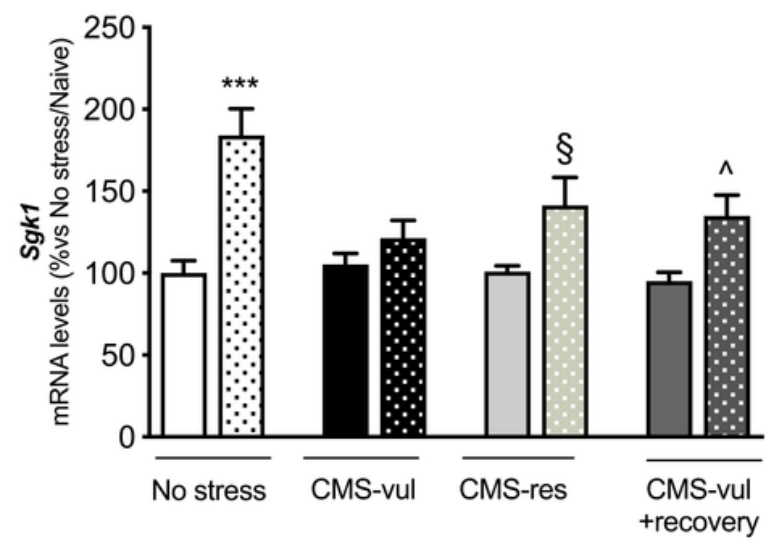

D

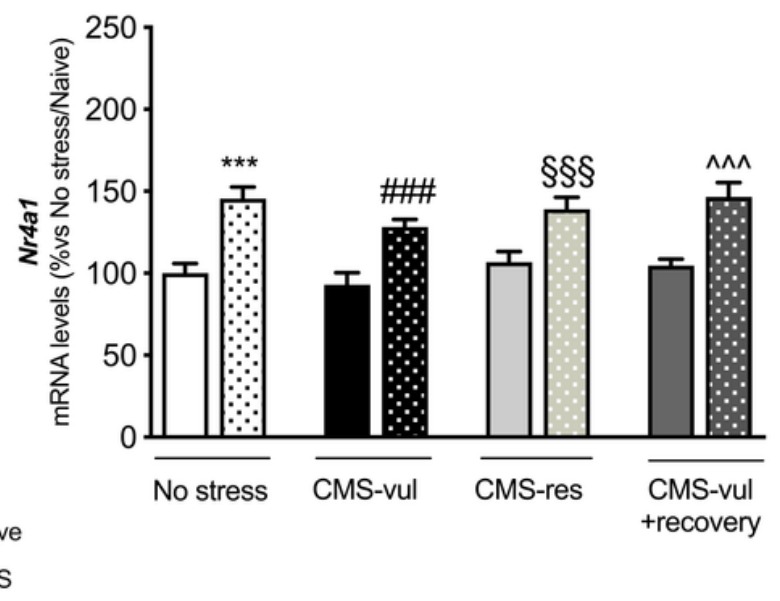

Figure 4

Analysis of Gadd45b, Sgk1, Dusp1 and Nr4a1 mRNA levels in the ventral hippocampus (vHip) of chronically stressed rats exposed to 1 hour of acute restraint stress (ARS).

The data are the mean \pm SEM: ${ }^{* \star} p<0.001$ vs No stress/Naive; ${ }^{\# \# \# ~} p<0.001$ vs CMS-vul/Naive; $\S p<0.05, \S \S \S p<0.001$ vs CMS-res/Naive; ${ }^{\wedge}<<0.05,{ }^{\wedge \wedge} p<0.001$ vs CMS-vul+recovery/Naive) (two-way ANOVA, Fisher's PLSD). 
IEGs
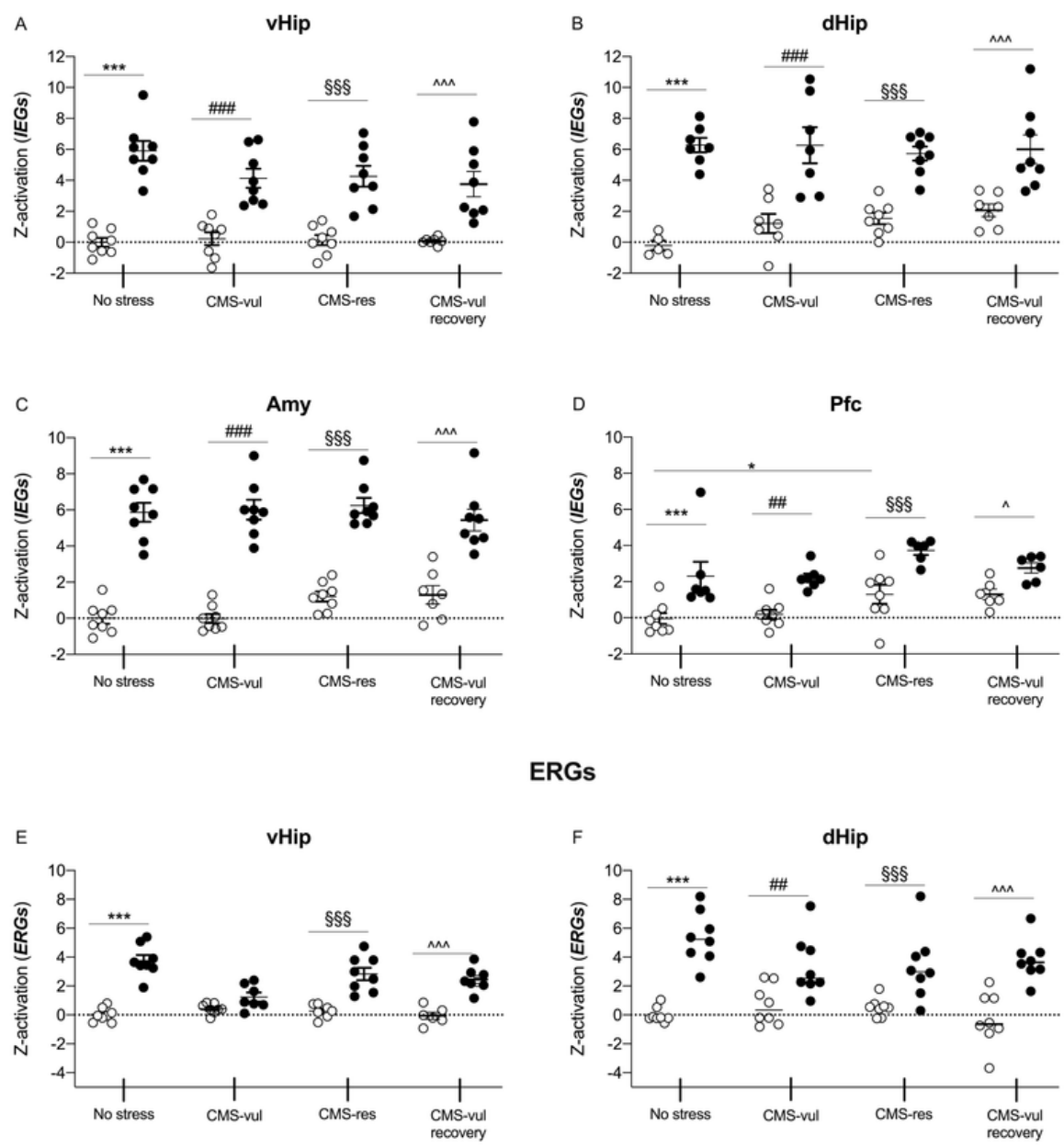

ERGs
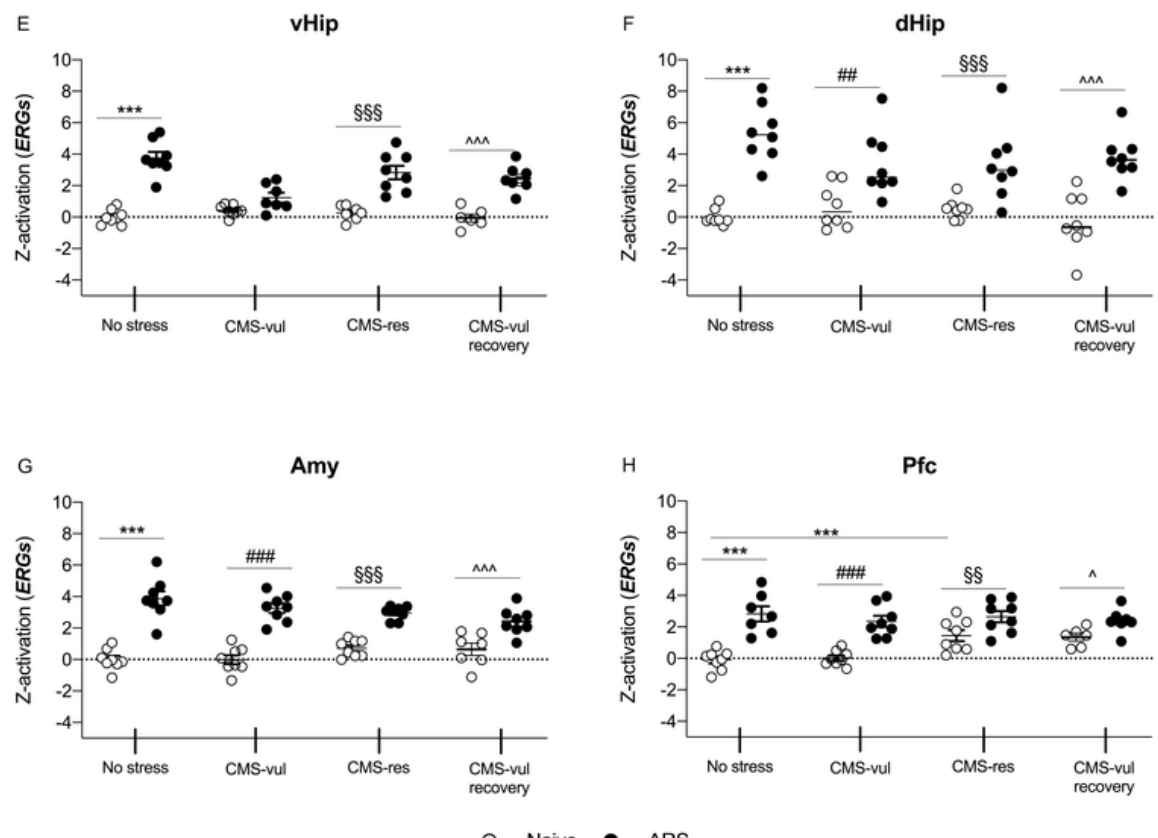

Figure 5

Z score activation of the IEGs and ERGs in the ventral hippocampus (vHip) (A-E), dorsal hippocampus (dHip) (B-F), amygdala (Amy) (C-G) and prefrontal cortex (Pfc) (D-H) of chronically stressed rats exposed to 1 hour of acute restraint stress (ARS).

The data are the mean \pm SEM: ${ }^{*} p<0.05,{ }^{* \star \star} p<0.001$ vs No stress/Naive; ${ }^{\# \#} p<0.01,{ }^{\# \# \#} p<0.001$ vs CMS-vul/Naive; $\S \S$ $p<0.01$, $\S \S \S p<0.001$ vs CMS-res/Naive; ${ }^{\wedge} p<0.05,{ }^{\wedge \wedge} p<0.001$ vs CMS-vul+recovery/Naive) (two-way ANOVA, Fisher's PLSD). 

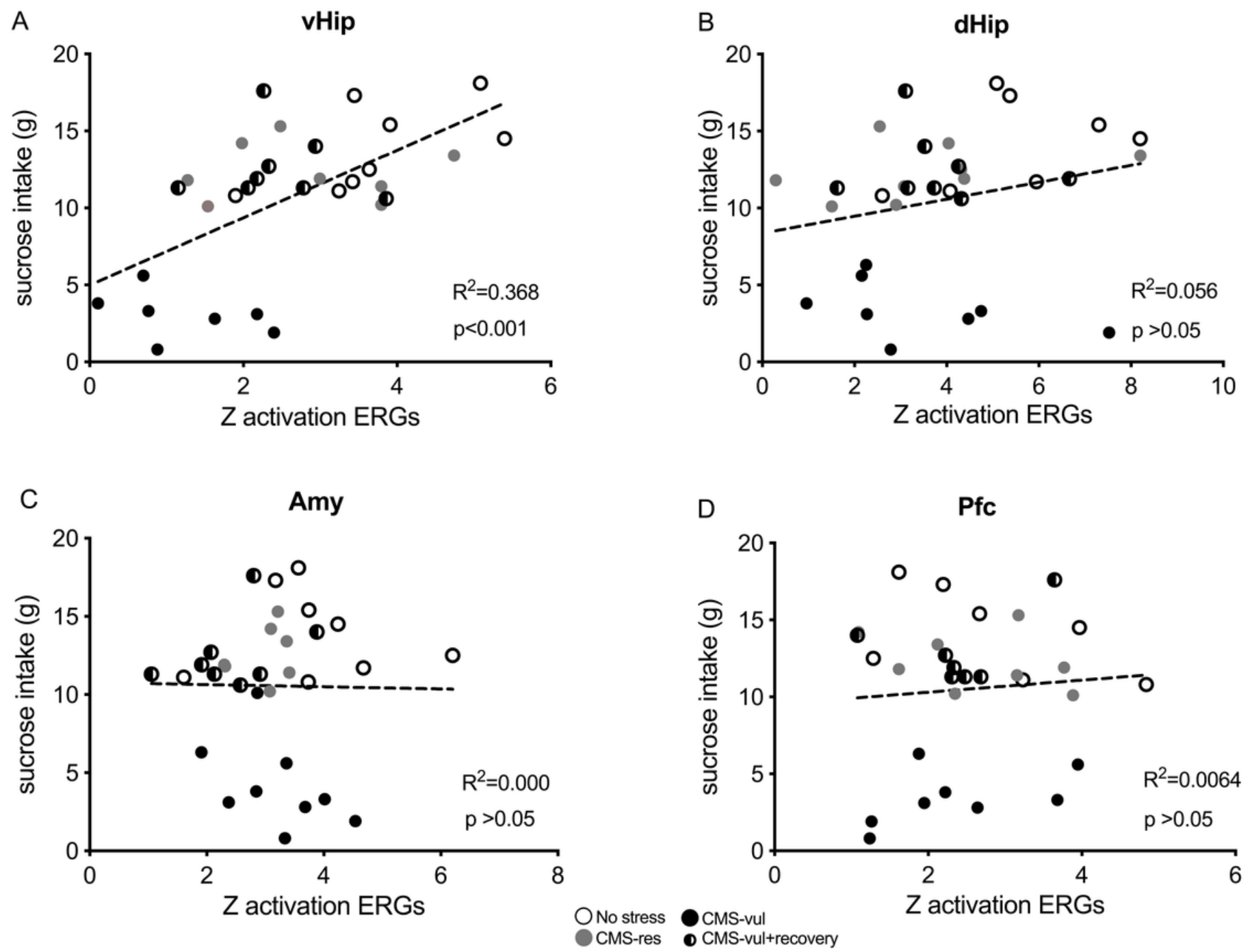

Figure 6

Pearson's product-moment correlation $(R)$ analysis between the sucrose intake and the $Z$ activation of the ERGs in $\operatorname{vHip}(A)$, dHip (B), Amy (C) and Pfc (D).

\section{Supplementary Files}

This is a list of supplementary files associated with this preprint. Click to download.

- supplementary.docx 